\title{
Analysis and Calculation for Conductive wheel precision of Electrochemical Grinding
}

\author{
Zhang Liaoyuan $^{1, \mathrm{a}}$, Meng Fanjiang ${ }^{1, \mathrm{~b}}$, Ji Zhenming ${ }^{1}$, Lv Tingting $^{1}$, Jiang Ruiqi $^{1}$ \\ ${ }^{1}$ Department of Mechanical Manufacturing and Automation, Shenyang Ligong University, Shenyang, \\ 110159, China \\ aemail: zly6217@163.com, bemail:517307951@qq.com
}

Keywords: Electrochemical making; Conductive wheel; CMM; Precision

\begin{abstract}
The machining accuracy of conductive is one of the important parameters which can affect the quality in the process of electrochemical ginding. In fact, there are many deviation in the machining process, and used the theory mathematical model, the two parts make a combination, we can take a model of factual grinding wheel in machining process, and discussed how many changed about affect part quality.
\end{abstract}

\section{Introduction}

The electrochemical machining(ECM),it's earliest used in grinding carbide. At that time, the equipment is expensive, and complex machine maintenance, or its development speed is slowly, so it has not been widely used. But as a result of comparison, this method possesses good leveling, grinding forces and grinding heat is small, no burns and crack surface, so there are any advantage in some areas.In recent years, used the pluse power of ECM to improve its processing effect. But for the quality of the original tool accuracy study, we will research the cutting tool machining precision in preliminary.

\section{Electrochemical principle of grinding}

That is electrochemical grinding system in fig.1. The 3 connect the anode, In the anode the clearance between the grinding wheel 3 and 2 bronze continuously bubbled into electrochemical grinding fluid, Produce electrochemical reaction between the workpiece and the cathode, After grinding wheel surface of the metal binding agent is an electrochemical dissolution of iron ions. By dissolution of iron ions in the surface of grinding wheel and insulating oxide film formation, The membrane thickness has a direct effect on electrical conductivity of the electrochemical process, Grinding wheel grinding surface must to maintain the proper amount of abrasive removal. Because of oxidation film as dielectric medium film, Make current is reduced quickly, and prevent electrochemical reaction to continue. As the grinding process, Thin film on the casting is move or thin, and reduce resistance to a certain value, The electrochemical reaction again. If in the process of grinding wheel and shaft concentricity of machine assembly is insufficient, or the state of the eccentric, it Will reduce the life of the grinding wheel and the quality of the processed parts in the process of grinding Wheel structure design.
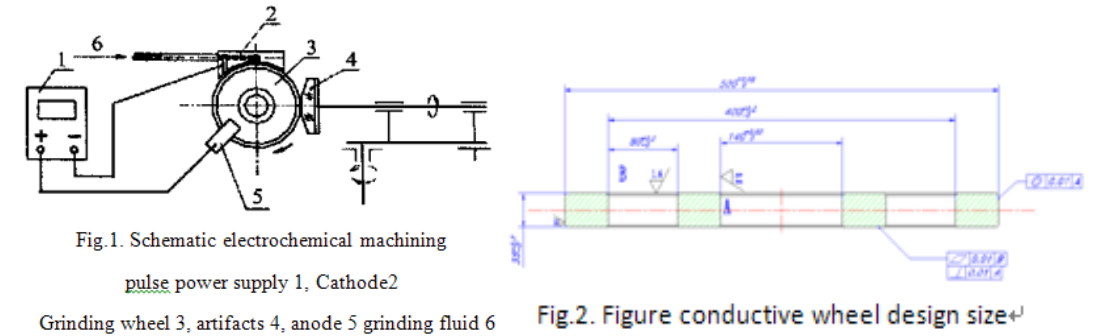

The principle of electrochemical machining, processing equipment analysis, mainly controlled the quality of the grinding wheel will be from the following two aspects:

Grinding wheel's coaxiality: With the existing processing capacity, parallel to axis of the bore axis of the outer ring which is the wheel in the course working. Achieve concentric theory is so 
difficult, so they would be controlled to within a reasonable range, as shown below fig, 2 .

Roughness of grinding wheel surface: On the surface of the grinding wheel needs to be plated, the thickness of the coating is about 3um, precision grinding wheel surface quality is bound to affect the use of the grinding wheel. And if the wheel's roughness is so bad, it will lead wheel plating surface irregularity, reduce the processing quality of the product during the electrochemical machining.

\begin{tabular}{ccccc}
\hline Name & $X$ & $\gamma$ & $Z$ & $D$ \\
\hline Inner Diameter & 0.021 .1 & 0.092 .1 & -34.965 .1 & 140.114 .1 \\
Outer Diameter & 0.021 .1 & 0.092 .1 & -34.965 .1 & 500.065 .1 \\
\hline
\end{tabular}

Table 1 conductive outer diameter and position of the wheel

\section{Coordinate size were detected wheel machining}

Use the CMM of FUNCTION, which is detection accuracy of $0.4 \mu \mathrm{m}$, and to achieve the principle of one tenth of conventional detection. Detecting the size of the key, the inner diameter of the grinding wheel in the design process, the outer diameter of the coaxial degree size is very important, which must be focus on it.

\section{Inner and outer diameter}

First used to select a coordinate plane, such as a B plane and then in a pore diameter $\varphi 140$ three or more randomly selected points of the point taken projected onto a plane, it is determined which one of the inner and outer diameter dimensions, such as round B1; Similarly, we take 3 points on the outside diameter of $\varphi 500$, such as cylindrical one. And we take another 3 points in the B flat plane of symmetry.

\section{Concentricity}

Usually, choosing the best way in the CMM, but that don't say know the part is ok, so we must choose another way, means the concentricity is less than the maximum $0.01 \mathrm{~mm}$.

$$
\begin{aligned}
& \tan \alpha=\frac{h}{L}=\frac{0.01}{35} \quad \alpha \leq 0.0164^{\circ} \\
& \tan \alpha_{T}=\sqrt{\triangle x^{2}-\triangle y^{2}}=0.000265 \quad \alpha_{T}=0.0152^{\circ}
\end{aligned}
$$

From the data, we know the actual manufacture of concentricity angle of 0.0152 , about $0.0093 \mathrm{~mm}$, and the concentricity of theory is $0.01 \mathrm{~mm}$. So, the size of the conductive grinding wheel is ok.
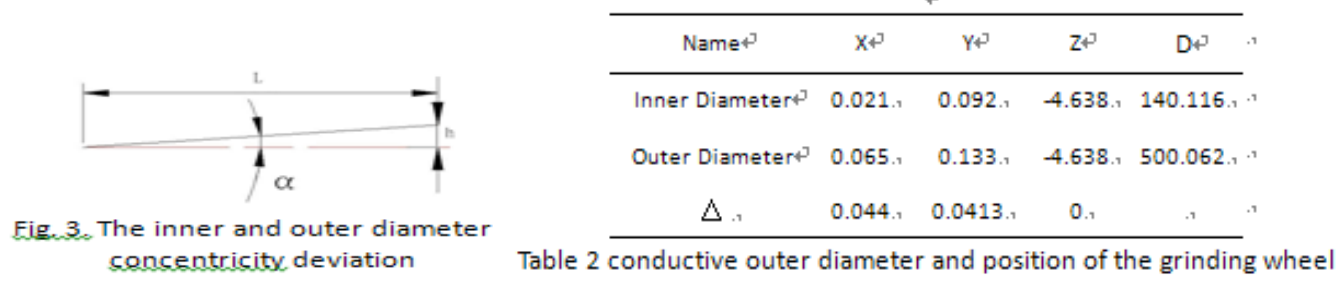

\section{Geometric accuracy of electrochemical grinding wheel arc length calculation}

The $\theta$ means point $\mathrm{A}$ of angular displacement (degrees); the $\Phi$ means point angular displacement of B (degrees); the Va means speed of removal ; the Va means point A (mm); the Vb means removal rate of the point $B(\mathrm{~mm})$. When the table traversing speed is Vc, electrochemical grinding arc length is $\widehat{L}_{1}+\widehat{L}_{2}$. Take a point $\mathrm{A}$ on the $\widehat{L}_{1}$, and that the removal rate of the anode material is $\mathrm{Va}$, the point $\mathrm{A}$ corresponds to the $\mathrm{X}$ direction of the material erosion rate of $\mathrm{Vacos} \theta$, the $\mathrm{X}$ direction of the material erosion rate of $\operatorname{Vasin} \theta$. After the time point $\mathrm{A} t$, where the gap in the $\mathrm{X}$ direction will result in an increment $\mathrm{dx}$, the gap in the $\mathrm{Y}$ direction will produce an increment dy, as 
shown in Fig.4 Schematic model of chemical grinding theory.
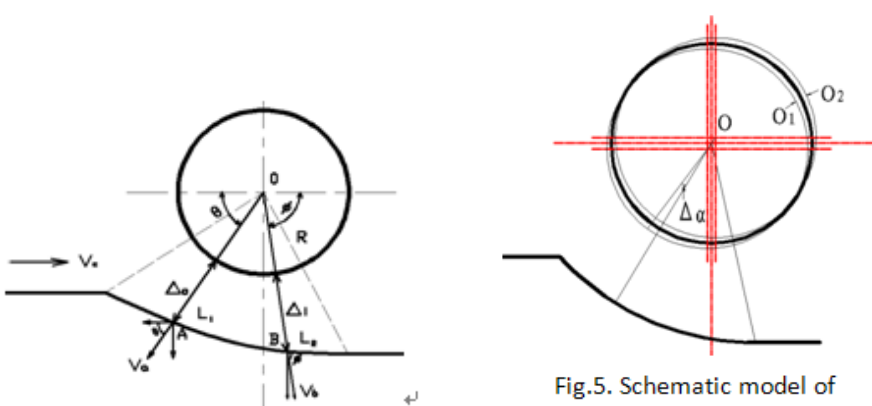

$d x=\left(V_{a} \cos \theta-V_{C}\right) d t$

Fig.4. Schematic model of chemical grinding theory

electrochemical grinding actual

$d \widehat{L}=\sqrt{d x^{2}+d y^{2}} \quad d x^{2}+d y^{2}=\left(V_{a}^{2}+V_{c}^{2}-2 V_{a} V_{c} \cos \theta\right) d t^{2}$

In general, the depth of the electrochemical grinding is relatively small, so that $\theta$ is very close to 90 degrees, it is desirable $\cos \theta=0$.

$$
\begin{aligned}
& d x^{2}+d y^{2}=\left(V_{a}^{2}+V_{c}^{2}\right) d t^{2} \\
& d \hat{L}_{1}=\sqrt{d x^{2}+d y^{2}}=\sqrt{V_{a}^{2}+V_{c}^{2}} d t=\left(\frac{\eta^{2} \omega^{2} \sigma_{1}^{2}}{2 \eta \omega \sigma U t+\Delta_{0}^{2}}+V_{c}^{2}\right)^{\frac{1}{2}} d t \\
& \hat{L}_{1}=\int_{0}^{\frac{R}{V_{c}}}\left(\frac{\eta^{2} \omega^{2} \sigma_{1}^{2}}{2 \eta \omega \sigma_{1} U t+\Delta_{0}^{2}}+V_{c}^{2}\right)^{\frac{1}{2}} d t
\end{aligned}
$$

For electrochemical arc $\widehat{L}_{2}$, the initial electrochemical gap $\Delta 1$ is the elapsed time t, $\widehat{L}_{1}$ electrochemical gap:

$$
\Delta_{1}=\sqrt{2 \rho \omega \sigma U t+\Delta_{0}^{2}}
$$

So that different gaps, electrochemical machining of different conductivity, the conductivity of $\sigma 2$. Located on any point of $B$, corresponding to a material that is corrosion erosion rate $\mathrm{Vb}$, the $\mathrm{X}$ direction corresponds to the point $\mathrm{A}$ on the removal rates of $\mathrm{Vbcos} \Phi$, the $\mathrm{X}$ direction of the erosion rate of $\mathrm{Vbsin} \Phi$. After point $\mathrm{B}$ the time $\mathrm{t}$, where the gap in the $\mathrm{X}$ direction will generate an increment $\mathrm{dx}$, dy in increments will produce a $\mathrm{Y}$ direction.

$$
\begin{aligned}
& d x=\left(V_{b} \cos \phi+V_{c}\right) d t \quad d y=V_{b} \sin \phi d t \\
& d \hat{L_{2}}=\sqrt{d x^{2}+d y^{2}}=\sqrt{V_{a}^{2}+V_{c}^{2}} d t=\left(\frac{\eta^{2} \omega^{2} \sigma_{2}^{2}}{2 \eta \omega \sigma_{2} U t+\Delta_{1}^{2}}+V_{c}^{2}\right)^{\frac{1}{2}} d t \\
& \hat{L}_{2}=\int_{0}^{t}\left(\frac{\eta^{2} \omega^{2} \sigma_{2}^{2}}{2 \eta \omega \sigma_{2} U t+\Delta_{1}^{2}}+V_{c}^{2}\right)^{\frac{1}{2}} d t
\end{aligned}
$$

Therefore, effective electrochemical grinding arc length,

$$
\hat{L}=\hat{L}_{1}+\hat{L}_{2}=\int_{0}^{\frac{R}{V_{c}}}\left(\frac{\eta^{2} \omega^{2} \sigma_{1}^{2}}{2 \eta \omega \sigma_{1} U t+\Delta_{0}^{2}}+V_{c}^{2}\right)^{\frac{1}{2}} d t+\int_{0}^{t}\left(\frac{\eta^{2} \omega^{2} \sigma_{2}^{2}}{2 \eta \omega \sigma_{2} U t+\Delta_{1}^{2}}+V_{c}^{2}\right)^{\frac{1}{2}} d t
$$

As shown in Figure 6, there is a machining error between the actual machining process, the actual center of the grinding wheel and the theoretical center of a certain offset, resulting in changes in the initial gap and the elapsed time $t$ after the occurrence of the gap, i.e.

$$
\begin{array}{ll}
\Delta_{0}(t)=\Delta_{0}+\Delta_{\max } \cos (\omega t+\phi) & \Delta_{1}(t)=\sqrt{2 \rho \omega \sigma U t+\Delta_{0}(t)^{2}} \\
\hat{L}=\hat{L_{1}}+\hat{L_{2}}=\int_{0}^{\frac{R}{V_{C}}}\left(\frac{\eta^{2} \omega^{2} \sigma_{1}^{2}}{2 \eta \omega \sigma_{1} U t+\Delta_{0}(t)^{2}}+V_{c}^{2}\right)^{\frac{1}{2}} d t+\int_{0}^{t}\left(\frac{\eta^{2} \omega^{2} \sigma_{2}^{2}}{2 \eta \omega \sigma_{2} U t+\Delta_{1}(t)^{2}}+V_{C}^{2}\right)^{\frac{1}{2}} d t
\end{array}
$$

Value $U_{t}=12 \mathrm{v}, \eta \omega=2.2, v_{a}=V_{c}=1 \mathrm{~mm} / \mathrm{min}, 7 \%-18 \%$ of the $\mathrm{NaCl}$ electrolytic solution, when $t=0$, Initial machining gap is $\Delta_{0 t}=0.8 \mathrm{~mm}$, so $\Delta_{1 t}=2.93 \mathrm{~mm}$, Electrochemical machining derived theoretical arc length is valid:

$$
\hat{L}=\hat{L}_{1}+\hat{L}_{2}=\int_{0}^{250}\left(\frac{(2.2 \times 0.15)^{2}}{2 \times 2.2 \times 0.15 \times 12 t+0.8^{2}}+1^{2}\right)^{\frac{1}{2}} d t+0=252.745 \mathrm{~mm}
$$

According to the entity conducting the largest wheel to get the actual processing, namely 


$$
\Delta_{0 \max }=\Delta_{0}+0.0093=0.8093 \mathrm{~mm} \quad \Delta_{1 \max }=\sqrt{2 \rho \omega \sigma U t+\Delta_{0 \max }(t)^{2}}=2.928 \mathrm{~mm}
$$

Similarly, when the effective electric arc grinding is:

$$
\hat{L}_{\operatorname{man}}=\hat{L}_{1}+\hat{L_{2}}=251.582 \mathrm{~mm} \quad \hat{L}_{\Delta}=\hat{L}+\hat{L}_{\max }^{\cap}=1.163 \mathrm{~mm}
$$

\section{Conclusion}

Thus, the actual processing of conductive grinding wheel contact arc has changed, for example the change of $\Delta_{0 \max } 、 \Delta_{0}$. When there is a deviation wheel size and position, effective arc conductive grinding wheel contact will periodically change. In this instance, when other electrochemical grinding process parameters unchanged, the position deviation when $0.0093 \mathrm{~mm}$, Effective arc deviation processing $\widehat{L}_{a}=1.163 \mathrm{~mm}$ Similarly, when the conductive grinding wheel in contact with the parts to be machined back,when $\Delta_{0 \min }=\Delta_{0}-0.093 \mathrm{~mm}, \Delta_{\Delta_{\min }}$ also decreases From the arc length formula, then processing the arc length becomes large.

\section{References}

[1]. Fan Zhishu, Li Xinzhong, Wang Tiancheng etc. Electrochemical machining and composite electrochemical machining [M]. Defense industry publication 2008.

[2]. Gong Qingshou, Zhu Qifan. Application of electrochemical finishing grinding [J] Hunan Institute of Engineering (Natural Science) 2001, 11 (3_4).

[3]. Zhang Liaoyuan. Machinery Industry Press, 2008.

[4]. Ren Xiaohong, Wang Jun.Electrochemical grinding centers [J] Microcomputer Information, 2009,16: 181-182 + 211 .

[5]. Zhang Liaoyuan, Wei Tao.Analysis of electrochemical grinding effective arc length and calculation [J] tool technology, 2013,02: 51-53.

[6]. Maehata H, Kamada H, and Yamamoto M. Electrolytic abra2sive mirror finishing Precision Engineering, 1987, 9 (1):31 43. 